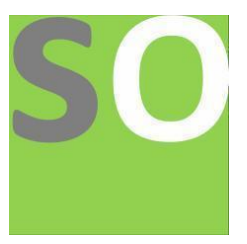

Article title: The Binary Goldbach Conjecture

Authors: Jan Feliksiak[1]

Affiliations: N. A.[1]

Orcid ids: 0000-0002-9388-1470[1]

Contact e-mail: jan.feliksiak1@yahoo.com

License information: This work has been published open access under Creative Commons Attribution License http://creativecommons.org/licenses/by/4.0/, which permits unrestricted use, distribution, and reproduction in any medium, provided the original work is properly cited. Conditions, terms of use and publishing policy can be found at https://www.scienceopen.com/.

Preprint statement: This article is a preprint and has not been peer-reviewed, under consideration and submitted to ScienceOpen Preprints for open peer review.

DOI: 10.14293/S2199-1006.1.SOR-.PPIU46T.v1

Preprint first posted online: 17 May 2021

Keywords: Goldbach conjecture, Binary Goldbach conjecture, Ternary Goldbach conjecture, Representation of even numbers as a difference of two primes, Primes in arithmetic progression, Prime Number Theorem PNT, Structure in primes 


\title{
THE BINARY GOLDBACH CONJECTURE
}

\author{
JAN FELIKSIAK
}

ABstract.

The Goldbach Conjecture, one of the oldest problems in mathematics, has fascinated and inspired many mathematicians for ages. In 1742 German mathematician Christian Goldbach, in a letter addressed to Leonhard Euler, proposed a conjecture. The modern day version of the Binary/Strong Goldbach conjecture asserts that:

Every even integer greater than 2 can be written as the sum of two primes.

The conjecture had been verified empirically up to $4 \times 10^{18}$, its proof however remains elusive, which seems to confirm that:

Some problems in mathematics remain buried deep in the catacombs of slow progress ... mind stretching mysteries await to be discovered beyond the boundaries of former thought.

Avery Carr (2013)

The research was aimed at exposition, of the intricate structure of the fabric of the Goldbach Conjecture problem. The research methodology explores a number of topics, before the definite proof of the Goldbach Conjecture can be presented. The Ternary Goldbach Conjecture Corollary follows the proof of the Binary Goldbach Conjecture as well as the representation of even numbers by the difference of two primes Corollary.

The research demonstrates that the Goldbach Conjecture is a genuine arithmetical question.

\section{(C)2019 Jan Feliksiak}

2010 Mathematics Subject Classification. 0102, 1102, 1103, 11A41, 11N05.

Key words and phrases. Goldbach conjecture, Binary Goldbach conjecture, Ternary Goldbach conjecture, sum of primes, primes in arithmetic progression, Prime Number Theorem. 


\section{INTRODUCTION}

Christian Goldbach in his letter to Leonhard Euler dated 7 June 1742, stated that every even number greater than 2 can be written as a sum of two prime numbers:

$$
2 m=p_{i}+p_{j} \quad \forall m \in \mathbb{N} \mid m \geq 2, \text { and prime numbers } p_{i}, p_{j} \in \mathbb{N} \mid p_{i} \leq p_{j}
$$

At the Second International Congress of Mathematicians in Paris, in August 1900, David Hilbert proposed a list of 23 mathematical problems, which he defined as problems of "deep significance for advancement of the mathematical science".

A great problem must be clear, because, what is clear and easily comprehended attracts, the complicated repels us ... It should be difficult in order to entice us, yet not completely inaccessible, lest it mock our efforts.

David Hilbert

Hilbert placed the Goldbach Conjecture, together with the Riemann Hypothesis and the Twin Primes Conjecture, as the 8-th problem on the list. The Goldbach Conjecture itself consists of two parts, the Binary (or Strong) Goldbach Conjecture and the Ternary (or Weak) Goldbach Conjecture. Although a massive effort has been exerted, yet both of them remain unproven. Many mathematicians worked on this problem, notably Brun [1], Hardy and Littlewood [14], Vinogradov [26], Chen [4], Kaniecki [16], Deshouillers et al [5] to name a few. Many more of the distinguished works are presented and/or referred to by Wang [27].

The Goldbach conjecture has even been a theme of a storybook written by Apostolos Doxiadis, under the title "Uncle Petros and Goldbach's Conjecture" [6]. The book tells the story of a man who dedicated his life to the research of the Goldbach Conjecture, searching for its proof. The publisher even offered a prize of 1 million dollars for a proof, as a part of a publicity stunt.

\section{The Binomial EXPANSION $2^{\left(n+\mathcal{G}_{(n)}\right)}$}

Within the scope of the paper, prime gap of the size $\mathfrak{g} \in \mathbb{N} \mid \mathfrak{g} \geq 2$ is defined as an interval between two primes $p_{i}, p_{i+1}$, containing $(\mathfrak{g}-1)$ composite integers. Maximal prime gap of the size $\mathfrak{g}$, is a gap strictly exceeding in size any preceding gap. All calculations and graphing were carried out with the aid of Mathematica ${ }^{\circledR}$ software.

One of the step-stones in this paper, is the bound on the maximal prime gaps. This part presented here, is an extract from a paper by Feliksiak [9]. For all $n \in \mathbb{N} \mid n \geq 5$, we make the following definitions:

Definition 2.1 (Scaling factor). $\xi=\frac{\log _{10}\left(\frac{n}{24}\right)}{\log _{10}(24)}$

Definition 2.2 (Interval length). $c=\mathcal{G}_{(n)}=\left\lfloor 5\left(\log _{10} n\right)^{2}\right\rfloor$

Definition 2.3 (Interval endpoint). $t=(n+c)$ 
Definition 2.4 (Binomial coefficient).

$$
\mathcal{M}_{(t)}=\left(\begin{array}{c}
n+c \\
n
\end{array}\right)=\left(\frac{(n+c) !}{(n ! c !)}\right)
$$

Definition 2.5 (Logarithm of the binomial coefficient).

$\log \mathcal{M}_{(t)}=\log \left(\frac{(n+c) !}{(n ! \times c !)}\right)=\log (t !)-\log (n !)-\log (c !)=\sum_{k=1}^{c} \log (n+k)-\sum_{k=1}^{c} \log k$

\subsection{Bounds on the logarithm of the binomial coefficient.}

Lemma 2.6 (Upper and Lower bounds on the log of $n !$ ).

The bounds on the logarithm of $n$ ! are given by:

$$
n \log (n)-n+1 \leq \log (n !) \leq(n+1) \log (n+1)-n \quad \forall n \in \mathbb{N} \mid n \geq 5
$$

Proof.

Evidently,

$$
\log (n !)=\sum_{k=1}^{n} \log (k) \quad \forall n \in \mathbb{N} \mid n \geq 2
$$

Now, the pertinent integrals to consider are:

$$
\int_{1}^{n} \log (x) d x \leq \log (n !) \leq \int_{0}^{n} \log (x+1) d x \quad \forall n \in \mathbb{N} \mid n \geq 5
$$

Accordingly, evaluating those integrals we obtain:

$$
\begin{aligned}
n \log (n)-n+1 \leq \log (n !) \leq n \log \left(\frac{(n+1)}{e}\right)+ & \log \left(\frac{(n+1)}{e}\right)+1 \\
& =(n+1) \log (n+1)-n
\end{aligned}
$$

Concluding the proof of Lemma 2.6.

\section{Remark 2.1.}

Observe that $\log \mathcal{M}_{(t)}$ is a difference of logarithms of factorial terms:

$$
\log \mathcal{M}_{(t)}=(\log (t !)-\log (n !)-\log (c !))
$$

Consequently, implementing the lower/upper bounds on the logarithm of $n$ ! for the bounds on $\log \mathcal{M}_{(t)}$, results in bounds of the form:

$$
\log \left(\frac{(t+k)^{(t+k)}}{(n+k)^{(n+k)}(c+k)^{(c+k)}}\right) \quad \text { for } \forall k \in \mathbb{N} \cup\{0\}
$$

Keeping the values of $c, n$ and $t$ constant and letting the variable $k$ to increase unboundedly, results in an unbounded monotonically decreasing function. When implementing the lower/upper bounds on the logarithm of $n$ ! for the Supremum/Infimum bounds on $\log \mathcal{M}_{(t)}$, the variable $k$ appears only with values $k=\{0,1\}$ respectively. The combined effect of the difference of the logarithms of factorial terms in $\log \mathcal{M}_{(t)}$ and the decreasing property of the function 2.5, imposes a reciprocal interchange of the bounds 2.1, when implementing them for the bounds on $\log \mathcal{M}_{(t)}$. 
Lemma $2.7\left(\log \mathcal{M}_{(t)}\right.$ Supremum Bound). by:

The Supremum Bound on the logarithm of the binomial coefficient $\mathcal{M}_{(t)}$ is given

$$
\log \mathcal{M}_{(t)} \leq \log \left(\frac{t^{t}}{n^{n} c^{c}}\right)-1=\mathcal{U B}_{(t)} \quad \forall n \in \mathbb{N} \mid n \geq 5
$$

Proof.

Evidently, by Lemma 2.6 we have:

$$
(n \log (n)-n+1) \leq \log (n !)
$$

Substituting from the inequality 2.7 into the Definition 2.5 we obtain:

$$
\begin{aligned}
& (\log (t !)-\log (n !)-\log (c !)) \\
& \leq((t \log (t)-t+1)-(n \log (n)-n+1)-(c \log (c)-c+1)) \\
& =t \log (t)-n \log (n)-c \log (c)-1=\log \left(\frac{t^{t}}{n^{n} c^{c}}\right)-1
\end{aligned}
$$

Consequently,

$$
\log \mathcal{M}_{(t)} \leq \log \left(\frac{t^{t}}{n^{n} c^{c}}\right)-1=\mathcal{U} \mathcal{B}_{(t)}
$$

The Supremum bound $\mathcal{U} \mathcal{B}_{(t)}$ produces an increasing, strictly monotone sequence in $\mathbb{R}$. At $n=5$, the difference $\mathcal{U} \mathcal{B}_{(t)}-\log \mathcal{M}_{(t)}$ attains 0.143365 and diverges as $n \rightarrow \infty$. Therefore, Lemma 2.7 holds as specified.

Lemma $2.8\left(\log \mathcal{M}_{(t)}\right.$ Infimum bound).

The Infimum Bound on the natural logarithm of the binomial coefficient $\mathcal{M}_{(t)}$ for all $n \in \mathbb{N} \mid n \geq 5$ is given by:

$$
\log \mathcal{M}_{(t)} \geq \log \left(\frac{(t+1)^{(t+1)}}{(n+1)^{(n+1)}(c+1)^{(c+1)}}\right)=\mathcal{L B}_{(t)}
$$

Proof.

From Lemma 2.6 we have:

$$
\log (n !) \leq n \log (n+1)-n+\log (n+1)
$$

Substituting from the inequality 2.11 into the Definition 2.5 we obtain:

$$
\begin{aligned}
& (2.12) \quad(\log (t !)-\log (n !)-\log (c !)) \\
& \geq t \log (t+1)-n \log (n+1)-c \log (c+1)+\log (t+1)-\log (n+1)-\log (c+1)
\end{aligned}
$$

$$
=\log \left(\frac{(t+1)^{t}}{(n+1)^{n}(c+1)^{c}}\right)+\log \left(\frac{(t+1)}{(n+1)(c+1)}\right)
$$

Consequently,

$$
\log \mathcal{M}_{(t)} \geq \log \left(\frac{(t+1)^{(t+1)}}{(n+1)^{(n+1)}(c+1)^{(c+1)}}\right)=\mathcal{L B}_{(t)}
$$

The Infimum bound $\mathcal{L B}_{(t)}$ produces an increasing, strictly monotone sequence in $\mathbb{R}$. At $n=5$, the difference $\log \mathcal{M}_{(t)}-\mathcal{L B}_{(t)}$ attains 0.455384 and diverges as $n \rightarrow \infty$. Therefore, Lemma 2.8 holds as specified. 


\section{MAXIMAL PRIME GAPS}
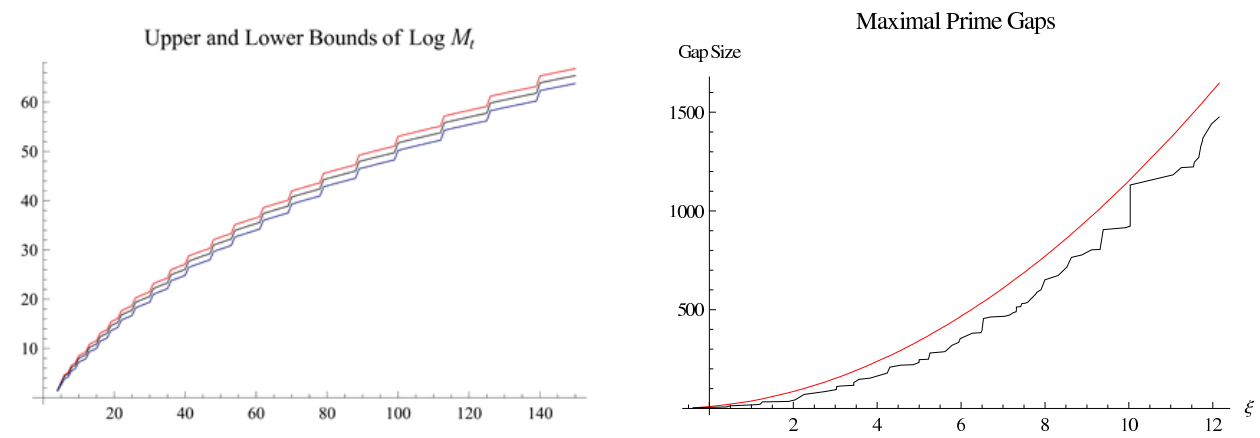

Figure 1. The left drawing shows the graphs of the lower (blue) and upper (red) bounds vs $\log \mathcal{M}_{(t)}$ (black). The right drawing shows the graph of $\mathcal{G}_{(n)}$ (red) and the actual maximal gaps (black) with respect to $\xi$ as given by the Definition 2.1. The graph has been produced on the basis of data obtained from C. Caldwell as well as from T. Nicely tables of maximal prime gaps.

We begin with a preliminary derivation. Since the integers from 1 to $\mathrm{n}$ contain $\left\lfloor\frac{n}{p}\right\rfloor$ multiples of the prime number $\mathrm{p},\left\lfloor\frac{n}{p^{2}}\right\rfloor$ multiples of $p^{2}$ etc. Thus it follows that:

$$
n !=\prod_{p} p^{u_{(n, p)}} ; \text { where } u_{(n, p)}=\sum_{m \geq 1}\left\lfloor\frac{n}{p^{m}}\right\rfloor
$$

In accordance with the definitions 2.2 of $\mathcal{G}_{(n)}, 2.3$ of $t$ and 2.4 of $\mathcal{M}_{(t)}$ we obtain:

$$
\mathcal{M}_{(t)}=\prod_{p \leq t} p^{\mathcal{K}_{p}}
$$

where

$$
\mathcal{K}_{p}=\sum_{m=1}^{\infty}\left(\left\lfloor\frac{t}{p^{m}}\right\rfloor-\left\lfloor\frac{n}{p^{m}}\right\rfloor-\left\lfloor\frac{\mathcal{G}_{(n)}}{p^{m}}\right\rfloor\right)
$$

it follows that

$$
\mathcal{K}_{p} \leq\left\lfloor\frac{\log t}{\log p}\right\rfloor
$$

and so by the above, Lemma 2.7 and 2.8 we have:

$$
\mathcal{L B}_{(t)} \leq \log \mathcal{M}_{(t)}=\log \prod_{p \leq t} p^{\mathcal{K}_{p}}=\sum_{p \leq t} \mathcal{K}_{p} \log p \leq \mathcal{U} \mathcal{B}_{(t)} \quad \forall n \in \mathbb{N} \mid n \geq 5
$$

Where $\mathrm{p}$ is as usual a prime number.

Lemma 3.1 (Prime Factors of $\mathcal{M}_{(t)}$ ).

The case when there does not exist any prime factor $p$ of $\mathcal{M}_{(t)}$ within the interval from $n$ to $\left(n+\mathcal{G}_{(n)}\right)=t$ for any $n \in \mathbb{N} \mid n \geq 8$, imposes an upper limit on all prime factors $p$ of $\mathcal{M}_{(t)}$. Consequently in this particular case, every prime factor $p$ must be less than or equal to $s=\left\lfloor\frac{t}{2}\right\rfloor$. 
Proof.

Let $\mathrm{p}$ be a prime factor of $\mathcal{M}_{(t)}$ so that $\mathcal{K}_{p} \geq 1$ and suppose that every prime factor $p \leq n$. If

$$
s<p \leq n
$$

then,

$$
p<\left(n+\mathcal{G}_{(n)}\right)<2 p
$$

and

$$
p^{2}>\left(\frac{\left(n+\mathcal{G}_{(n)}\right)}{2}\right)^{2}>\left(n+\mathcal{G}_{(n)}\right)
$$

and so $\mathcal{K}_{p}=0$. Therefore $p \leq s$ for every prime factor $\mathrm{p}$ of $\mathcal{M}_{(t)}$, for any $n \in \mathbb{N} \mid n \geq 8$.

\subsection{Maximal prime gaps upper bound.}

The binomial coefficient $\mathcal{M}_{(t)}$ :

$$
\begin{array}{r}
2^{t / 2}<n^{\frac{c}{2}}<\exp \left(\mathcal{L B}_{(t)}\right) \leq \mathcal{M}_{(t)}=\left(\frac{(n+c) !}{(n ! \times c !)}\right) \leq \exp \left(\mathcal{U B}_{(t)}\right)<n^{\frac{2 c}{3}}<2^{t} \\
\forall n \in \mathbb{N} \mid n \geq 22
\end{array}
$$

The bounds on the logarithm of $\mathcal{M}_{(t)}$ are given by Lemma 2.7 and 2.8:

$$
\begin{aligned}
& \mathcal{L B}_{(t)}=\log \left(\frac{(t+1)^{(t+1)}}{(n+1)^{(n+1)}(c+1)^{(c+1)}}\right) \\
& \leq \log \mathcal{M}_{(t)}=\sum_{k=1}^{c} \log (n+k)-\sum_{k=1}^{c} \log k \leq \log \left(\frac{t^{t}}{n^{n} c^{c}}\right)-1=\mathcal{U} \mathcal{B}_{(t)} \\
& \forall n \in \mathbb{N} \mid n \geq 5
\end{aligned}
$$

The proof of the Maximal Gaps Theorem implements the Supremum bound function $\mathcal{U} \mathcal{B}_{\left(t_{s}\right)}$. Due to the fact that the Supremum function $\mathcal{U} \mathcal{B}_{(t)}$ applies values of $n, c$ and $t$ directly, it imposes a technical requirement to generate a set of pertinent values, to correctly approximate the interval $s$. This is to ascertain that the generated interval is at least equal to $s$, and the corresponding value of $c$ is correct. Respective definitions follow:

Definition 3.2. $n_{s}=\frac{n}{2}$

Definition 3.3. $c_{s}=5\left(\log _{10}\left(n_{s}\right)\right)^{2}+1$

Definition 3.4. $t_{s}=n_{s}+c_{s}$

Theorem 3.5 (Maximal Prime Gaps Bound and Infimum for primes).

For any $n \in \mathbb{N} \mid n \geq 8$ there exists at least one $p \in \mathbb{N} \mid n<p \leq t$; where $p$ is as usual a prime number and the maximal prime gaps upper bound $\mathcal{G}_{(n)}$ is given by:

$$
\mathcal{G}_{(n)}=\left\lfloor 5\left(\log _{10} n\right)^{2}\right\rfloor \quad \forall n \in \mathbb{N} \mid n \geq 8
$$

$$
\text { Equivalently, } p_{i+1}-p_{i} \leq \mathcal{G}_{\left(p_{i}\right)}
$$


Proof.

Suppose that there is no prime within the interval from $n$ to $t$. Then in accordance with the hypothesis, by Lemma 3.1 we have that, every prime factor $p$ of $\mathcal{M}_{(t)}$ must be less than or at most equal to $s=\left\lfloor\frac{t}{2}\right\rfloor$. Invoking Definitions 3.2, 3.3 and 3.4, Lemma 2.7, 2.8 and the inequality 3.1 we derive for all $n \in \mathbb{N} \mid n \geq 8$ :

$$
\begin{aligned}
& \text { (3.4) } \mathcal{L B}_{(t)}=\log \left(\frac{(t+1)^{(t+1)}}{(n+1)^{(n+1)}(c+1)^{(c+1)}}\right) \\
& \leq \log \mathcal{M}_{(t)}=\log \prod_{p \leq t_{(s)}} p^{\mathcal{K}_{p}}=\sum_{p \leq t_{(s)}} \mathcal{K}_{p} \log p \leq \log \left(\frac{\left(t_{s}\right)^{t_{s}}}{\left(n_{s}\right)^{n_{s}}\left(c_{s}\right)^{c_{s}}}\right)-1=\mathcal{U} \mathcal{B}_{\left(t_{s}\right)}
\end{aligned}
$$

In accordance with the hypothesis therefore, it must be true that:

$$
a_{c}=\log \left(\frac{(t+1)^{(t+1)}}{(n+1)^{(n+1)}(c+1)^{(c+1)}}\right)-\log \left(\frac{\left(t_{s}\right)^{t_{s}}}{\left(n_{s}\right)^{n_{s}}\left(c_{s}\right)^{c_{s}}}\right)+1<0
$$

Now, we apply the Cauchy's Root Test for $n \geq 43$ :

$$
\lim _{c \rightarrow \infty} \sqrt[c]{\left|a_{c}\right|}=\lim _{c \rightarrow \infty} \sqrt[c]{\left|\mathcal{L B}_{(t)}-\mathcal{U} \mathcal{B}_{\left(t_{s}\right)}\right|} \rightarrow 1
$$

At $n=43$ the Cauchy's Root Test attains $\approx 1.17851$ and tends asymptotically to 1 , decreasing strictly from above. Thus, by the definition of the Cauchy's Root Test, the series formed from the terms of the difference $\mathcal{L B}_{(t)}-\mathcal{U} \mathcal{B}_{\left(t_{s}\right)}$, diverges as $c$ increases unboundedly. Hence, in accordance with the hypothesis, inequality 3.5 diverges to $-\infty$ as $n$ increases unboundedly. However, at $n=43$ the difference 3.5 attains $\sim 9.45885151$ and diverges as $n$ increases unboundedly. Hence, we have a contradiction to the initial hypothesis. This implies that for all $n \in \mathbb{N} \mid n \geq 43$ :

$$
\mathcal{L B}_{(t)}-\mathcal{U B}_{\left(t_{s}\right)}>0
$$

Necessarily therefore, there must be at least one prime within the interval $c$ for all $n \in \mathbb{N} \mid n \geq 43$. Table 1 lists all values of $n$ s.t. $8 \leq n \leq 47$. Evidently, every possible sub-interval contains at least one prime number. Thus we deduce that Theorem 3.5 holds in this range as well. Consequently Theorem 3.5 holds as stated for all $n \in \mathbb{N} \mid n \geq 8$. Thus completing the proof.

TABLE 1 . Low range $\mathcal{G}_{(n)}$ vs primes within the range

\begin{tabular}{|c|c|c|c|c|c|}
\hline$n$ & $\mathcal{G}_{(n)}$ & primes & $n$ & $\mathcal{G}_{(n)}$ & primes \\
\hline 8 & 4 & 11 & 29 & 10 & 31,37 \\
\hline 11 & 5 & 13 & 31 & 11 & 37,41 \\
\hline 13 & 6 & 17,19 & 37 & 12 & $41,43,47$ \\
\hline 17 & 7 & 19,23 & 41 & 13 & $43,47,53$ \\
\hline 19 & 8 & 23 & 43 & 13 & 47,53 \\
\hline 23 & 9 & 29,31 & 47 & 13 & 53,59 \\
\hline
\end{tabular}




\section{The Binary/Strong Goldbach Conjecture}

From the pragmatic point of view, we incorporate into the research methodology of the Goldbach Conjecture, the aspect of prime numbers in Arithmetic Progression (AP). We begin with the Theorem by P.G. Lejeune-Dirichlet (1837).

Theorem 4.1 (Primes in arithmetic progression).

Let $d$ and $a$ be co-prime integers. Then the arithmetic progression $a, a+d, a+$ $2 d, a+3 d, \ldots$ contains infinitely many primes. Moreover, the density of the set $D=\{p$ s.t. $\mathrm{p}$ is prime, $p \equiv a(\bmod d)\}$ in the set of primes is $\frac{1}{\phi d}$, where $\phi$ is the Euler totient function. Equivalently, the set $D$ has infinite cardinality.

For a proof, please consult pertinent literature. The natural numbers can be split into six disjoint equivalence classes, their union producing $\mathbb{N}$. Since prime numbers $p>2$ are odd, there are only three pertinent equivalence classes to consider. Let's define the set $\mathcal{S}$ :

\section{Definition 4.2 .}

$\mathcal{S}=\{s \mid s=6 n+3$, or $s=6 n+5$, or else $s=6 n+7\} \quad \forall n \in \mathbb{N} \cup\{0\}$

Clearly, $\mathcal{S}$ contains all odd natural numbers $s \geq 3$. Since for all $n \in \mathbb{N} \mid s=6 n+3$, the element $s \equiv 0(\bmod 3)$, consequently, this equivalence class produces composite numbers exclusively. The remaining two equivalence classes: $6 n+5$ and $6 n+7$, by Theorem 4.1 produce infinitely many primes each. Because all primes $p \in \mathbb{N} \mid p \geq 3$ are odd, this implies that $\mathcal{S}$ contains all prime numbers $p \geq 3$. Let's therefore define an acronym to represent the two equivalence classes:

Definition 4.3 (Lower Prime Form Integer). $L P F=\{6 n+5 \mid n \in \mathbb{N} \cup\{0\}\}$

Definition 4.4 (Upper Prime Form Integer). $U P F=\{6 n+7 \mid n \in \mathbb{N} \cup\{0\}\}$

Clearly, the union of $L P F \cup U P F$ equivalence classes and $\{2,3\}$ contains all primes $p \in \mathbb{N}$ (It also contains composite numbers as well).

The concept of adding two prime numbers, in order to obtain an even number, is well known. It is however a challenging and very intricate problem, a view also shared and verified by E. Calude in her research paper, Calude [2].

From the Binomial Theorem, we have that the number of possible combinations of pairs of primes $2 \leq p_{i}, p_{j} \leq p_{k}$, to form a sum is given by the Binomial Coefficient $C((k+1), 2)$ where the order does not count and repetitions are permitted:

$$
C_{k}=\frac{(k+1) !}{2 ! \times(k-1) !}=\frac{k(k+1)}{2}
$$

Obviously there will be repeats, not only with the two prime summands reversed, but also numerically, with different pairs of primes generating the same even number. In addition to that there will be combinations involving the prime number 2. Now, for prime numbers $p_{i}, p_{j}, p_{k} \in \mathbb{N}$, let's define the set (assumed already sorted and any existing repeats discarded):

Definition 4.5. $\mathcal{E}=\left\{e, e \in \mathbb{N} \mid e \equiv 0(\bmod 2), e=p_{i}+p_{j}\right.$, with $\left.p_{i}, p_{j} \leq p_{k}\right\}$ 
The generated set $\mathcal{E}$, contains all possible sums of two odd primes $p_{i}, p_{j} \leq p_{k}$ up to $2 p_{k}$. This set may only include new elements when an additional prime number will become available in the range.

A natural question arises, "will all of those generated distinct even numbers be consecutive?" In general, depending on $p_{k}$ and $e$, there will be values missing in the set $\mathcal{E}$. Let's define the function $N^{\circ}$, containing strictly the count of distinct, consecutive even numbers, elements of the set $\mathcal{E}$, strictly up to (not including), the first failed/skipped even number:

Definition 4.6. $N^{\circ}=\sum_{e} 1 \quad$ for $e \in \mathcal{E} \mid e=p_{i}+p_{j}$, with $p_{i}, p_{j} \leq p_{k}$

The function $N^{\circ}$ is weakly increasing, since it is only possible to generate new distinct, consecutive even numbers, with additional primes coming into consideration. Between the primes, the function $N^{\circ}$ exhibits a horizontal/level slope at the height of the count attained at the last failed/skipped even number. The expected exact count of distinct, consecutive even numbers in the range up to $p_{k}$, necessary to validate the Goldbach's Conjecture, clearly is:

$$
N^{\circ}=\left\lfloor\frac{p_{k}}{2}\right\rfloor-1 \quad 4 \leq p_{i}+p_{j}<p_{k}
$$

Since however, the greatest even integer that can be generated, by implementing primes $p_{i}, p_{j} \leq p_{k}$ is $2 p_{k}$, some of the generated even numbers will clearly exceed $p_{k}$. The prime $p_{k}$ itself will obviously recur in pairs with greater primes, this is however a secondary issue. The true count of the generated distinct, consecutive even numbers is contained within the range:

$$
3<N^{\circ} \leq p_{k}-1 ; \quad \text { for } 4 \leq p_{i}+p_{j} \leq 2 p_{k}
$$

Where the lower limit, represents the first 3 even numbers $4,6,8$, which can not be generated, by the sole use of the $\mathbf{A P}$ formulae and $p_{k}-1$ indicates, that we exclude the number 2 from consideration. When implementing the AP formulae, the sum of two arbitrary LPF and/or UPF integers for all $m, n \in \mathbb{N} \cup\{0\}$, takes the form:

- Case 1: The even number $e$ is all LPF variety. The sum of two arbitrary LPF integers produces an even integer of the form:

(4.4) $e=(6 m+5)+(6 n+5)=6(m+n+1)+4$, producing $\{10,16,22,28, \ldots\}$

- Case 2: The even number $e$ is a Mixed variety. The sum of an arbitrary LPF integer and an arbitrary UPF integer produces an even integer of the form:

$(4.5) e=(6 m+5)+(6 n+7)=6(m+n+1)+6$, producing $\{12,18,24,30, \ldots\}$

- Case 3: The even number $e$ is all UPF variety. The sum of two arbitrary UPF integers produces an even integer of the form:

$(4.6) e=(6 m+7)+(6 n+7)=6(m+n+1)+8$, producing $\{14,20,26,32, \ldots\}$

In the formulae 4.5 and 4.6 above, we could have inserted 0 and 2 respectively. However, by inserting 6 and 8 instead, we actually carry a bit of information that will be of assistance later. The exception here, is the case of the set of summands $\{3,(e-3)\}$. This particular set of summands can only be assigned to Case 1 or Case 3. An instance which depends on the variety of $e$. 

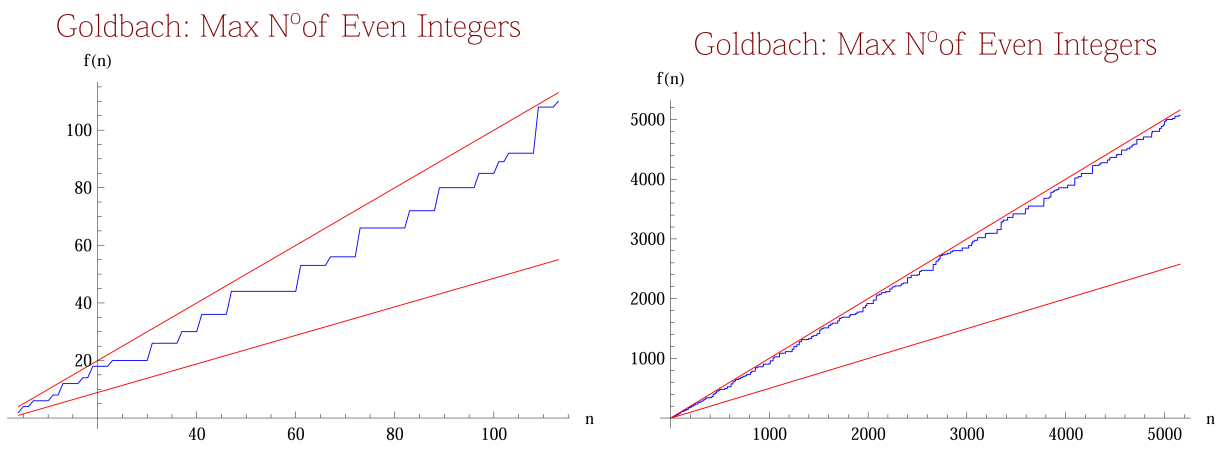

FiguRE 2. The drawing shows the graph of the function $N^{\circ}$ (Blue) displaying the maximal volume of distinct, consecutive even numbers generated in the range $4 \leq e \equiv 0(\bmod 2) \leq 2 n$ at every $n$ in the range. The lower line (Red) is $f_{n}=\left\lfloor\frac{n}{2}\right\rfloor-1$, while the top line (Red) is $f_{n}=n$. The L.H. figure is drawn in the range $4 \leq n \leq 113$. The R.H. figure is drawn in the range $4 \leq n \leq 5153$.

The number of elements of the set $\mathcal{E}$, containing the collection of all distinct even numbers $e$, subject to $e, p_{i}, p_{j} \in \mathbb{N} \mid e \equiv 0(\bmod 2), e=p_{i}+p_{j}, 2<p_{i}, p_{j} \leq p_{k}$, strictly increases with increasing number of primes considered. These generated even numbers however, will not always be consecutive. If $e<p_{k}$ then obviously, we will obtain solutions with $p_{i}, p_{j}<p_{k}$. If however, $p_{k}<e \leq 2 p_{k}$ in some pairs comprising the solution $e=p_{i}+p_{j}$, one of the primes say $p_{j}$, may and often will exceed $p_{k}$. The closer, some even number $e$ is to $2 p_{k}$, the greater the chance, that one of the summands will exceed $p_{k}$.

\section{Deterministic Procedure Generating the Solution Set}

We may apply a simple procedure to generate the full solution set for a particular even number $e$ :

Procedure 5.1 (Generating The Solution Set).

- Implement equations 4.4 through 4.6 above, to identify the particular variety, to which the even number $e$ belongs.

- Compute $e / 2$. If $e / 2$ is prime, we may stop here, or proceed further to generate the entire solution set.

- Find the nearest to the $e / 2$, LPF or UPF integers (as is appropriate), one on each side of $e / 2$. In the case of $e$ being of the Mixed Type, we have to run the procedure twice. We run the the procedure once with the LPF integer being located on the low side and the second time with the LPF integer being on the high side. The UPF integer of course will always be located on the opposite side.

- If both of the found integers are prime numbers, they constitute a valid solution, possibly one of a number of valid solutions.

- Next, decrease the lower integer by 6 and increase the higher integer by 6 . This constitutes another possible solution, providing that both are prime numbers. 
- Continue to decrease/increase by 6 the respective integers, until the lower limit of 5 or 7 is reached. Every pair generated this way, constitutes a solution. However, to form a valid solution, both integers must simultaneously be prime.

- Verify if $\{3, e-3\}$ constitutes a valid solution, if so, add it to the solution set.This solution is not categorized, because the prime number 3 is neither part of the AP LPF, nor UPF variety. The only AP LPF or UPF variety is $e-3$ itself.

The procedure terminates upon reaching the predefined sentinel $\{5,7, e\}$ at the low or the high end, or both. In general, over the entire range of $2 p_{k}$, there are two possible scenarios:

(1) Case A: All distinct, consecutive even numbers up to and including $n=2 p_{k}$ were generated successfully. This implies that the function $N^{\circ}=p_{k}-1$ (we obtain one even number less, because $2 \neq p_{i}+p_{j}$ ). Case A however, is an exception only.

(2) Case B: In general, the smallest even number $e \in \mathbb{N}$ is found, which failed to be generated by the entire set $\left\{p_{i}, p_{j} \in \mathbb{N} \mid p_{i}, p_{j} \leq p_{k}\right\}$. The solution can be found by implementing the Procedure 5.1.

Case A. Due to the fact that the maximal gaps upper bound is given by Theorem 3.5:

$$
\begin{array}{r}
\mathcal{U B}=\left\lfloor 5\left(\log _{10} p_{k}\right)^{2}\right\rfloor \quad \forall p_{k} \in \mathbb{N} \mid p_{k} \geq 11 \\
\text { Equivalently, } p_{(k+1)}-p_{k} \leq \mathcal{U B}
\end{array}
$$

From inequality 5.1 we have that:

$$
p_{(k+1)}<\mathcal{U B}+p_{k}
$$

and clearly,

$$
\mathcal{U B} \ll p_{k}
$$

Obviously therefore, we have that:

$$
p_{(k+1)}<2 p_{k}
$$

Consequently, we see that we can generate all even numbers less than $p_{(k+1)}$ and more, up to and including $2 p_{k}$. Five low $p_{k}$ range cases are: $\{5,7,13,19,109\}$.

Case B. Suppose that some even number $e \in \mathbb{N}$ exists, such that $p_{k}<e<2 p_{k}$, which cannot be expressed as a sum $e=p_{i}+p_{j} \mid p_{i}, p_{j} \leq p_{k}$. We only need to be concerned with the smallest such number $e$. This is because, once the sequence of distinct and consecutive even integers is broken, it is impossible to generate this particular even number $e$, due to the fact that it does not have summands that both simultaneously $p_{i}, p_{j} \leq p_{k}$. Such summands constitute a legitimate solution, which cannot be reconciled with a failed generation of this number. At the very least it is a prime number $p=p_{(k+1)}$.

This even number $e$ immediately terminates the generated sequence of distinct, consecutive even numbers. All other even numbers, which possibly continue intermittently from this point on, up to the upper limit of $2 p_{k}$, are not considered in this document. 
This number establishes a clear limit on the distinct, consecutive even numbers generated $e=p_{i}+p_{j} \mid p_{i}, p_{j} \leq p_{k}$. This will only change, after we reach the prime $p_{(k+c)}$ which actually is one of the summands of that number $e$. This implies, that such limit will possibly persist over a range of prime numbers $p$.

Clearly, the function $N^{\circ}$ remains constant in such a case until $p_{(k+c)}$. Consequently, the graph of the function $N^{\circ}$ exhibits a horizontal slope within such an interval. Therefore, by the definition $N^{\circ}$ is a weakly increasing function.

Now obviously, $e / 2 \leq p_{k}<p_{(k+1)}$. By Theorem 3.5, we have that $p_{(k+1)}<2 p_{k}$. Since the set of primes $\left\{2,3,5,7, \ldots, p_{k}\right\}$, does not form a solution $e=p_{i}+p_{j}$ we advance implementing the Procedure 5.1. This implies that the next possible candidate for a valid summand (w.l.o.g. say $p_{j}$ ) in the range $p_{k}<p_{j}<e$ is $p_{(k+1)}$. This prime number must of course conform to the class variety code of $e$ (equations 4.4 through 4.6$)$.

This implies that the summand $p_{j} \geq p_{(k+1)}$. Since $p_{j} \geq p_{(k+1)}$, lets denote $p_{j}=p_{(k+c)} \mid c \in \mathbb{N}$ in general. Then, the even number equals:

$$
e=p_{(k+c)}+p_{i}
$$

this implies that,

$$
p_{(k+1)}<e<2 p_{k}
$$

We can arrange the equations 4.4 through 4.6 , to show their alignment:

$$
\begin{aligned}
6\left(m_{1}+n_{1}+1\right)+4<6\left(m_{1}\right. & \left.+n_{1}+1\right)+5<6\left(m_{2}+n_{2}+1\right)+6 \\
& <6\left(m_{3}+n_{3}+1\right)+7<6\left(m_{3}+n_{3}+1\right)+8
\end{aligned}
$$

Frequently, some of the distinct variables $m_{i}$, or $n_{i}$, will have the same numerical value. Referring however, to equations 4.4 to 4.6 above, we see that depending on $e$, they will originate from different variety of primes.

\subsection{Examples.}

\section{Example 5.2.}

This example is analyzed carefully, providing all solutions. The number $e=224$ can not be formed by any combination of primes $p_{i}, p_{j} \leq p_{k}=113 \mid p_{i}+p_{j}=224$. This even number clearly is within the range of $2 p_{k}=226$, however it requires a prime for one of the summands, which at that point is out of range. Consequently, the graph of the function $N^{\circ}$ exhibits a horizontal slope in the range $113 \rightarrow 127$ at the height of 108.

The complete solution for the even number $e=224$ is presented in tabular form. We implement the Procedure 5.1. This even number belongs to Case 3, which means that all UPF solution is required. Now, $224 / 2=112$, hence we begin to search for possible UPF candidates at 112. The nearest lower candidate is 109, the nearest higher candidate is 115 . Both are solutions to 224 , however one of the summands is composite. Hence we proceed further decreasing/increasing the numbers. The results are listed in Table 3. 
TABLE 2. Summands for $e$ within the range $112 \rightarrow 128$

\begin{tabular}{|c|c|c|}
\hline Even number & Summands & $e$ variety \\
\hline 112 & 5,107 & LPF \\
\hline 114 & 5,109 & Mixed \\
\hline 116 & 7,109 & UPF \\
\hline 118 & 59,59 & LPF \\
\hline 120 & 7,113 & Mixed \\
\hline 122 & 61,61 & UPF \\
\hline 124 & 11,113 & LPF \\
\hline 126 & 13,113 & Mixed \\
\hline 128 & 19,109 & UPF \\
\hline
\end{tabular}

TABLE 3. Summands comprising the solution of $p_{i}+p_{j}=224$

\begin{tabular}{|c|c|}
\hline Summands & Solution type \\
\hline 109,115 & Invalid \\
\hline 103,121 & Invalid \\
\hline 97,127 & Valid \\
\hline 91,133 & Invalid \\
\hline 85,139 & Invalid \\
\hline 79,145 & Invalid \\
\hline 73,151 & Valid \\
\hline 67,157 & Valid \\
\hline 61,163 & Valid \\
\hline 55,169 & Invalid \\
\hline 49,175 & Invalid \\
\hline 43,181 & Valid \\
\hline 37,187 & Invalid \\
\hline 31,193 & Valid \\
\hline 25,199 & Invalid \\
\hline 19,205 & Invalid \\
\hline 13,211 & Valid \\
\hline 7,217 & Invalid \\
\hline
\end{tabular}

\section{Example 5.3.}

The even number $e=4952$, cannot be formed from the set of primes $3, \ldots, 2539$. The upper limit for this prime is $2 \times 2539=5078$, hence, 4952 is well within this range. This particular even number $e$, constitutes Case 3, all UPF primes solution. Searching systematically, we find that $e=4952=2293+2659$. The prime 2659 , is the 6-th UPF prime after the prime 2539. The full set is: $\{2539,2543,2549,2551,2557,2579,2591,2593,2609,2617,2621,2633,2647,2657,2659\}$ The LPF primes are in Black, while the UPF primes are in Red. 


\section{Resolution of the Goldbach Conjecture}

We begin with some definitions and preliminary derivations.

Definition 6.1 (Interval length).

$$
c=5 \sqrt{p_{k}} \quad \forall p_{k} \in \mathbb{N} \mid p_{k} \geq 5
$$

Definition 6.2 (Interval endpoint).

$$
t=\left(p_{k}+c\right)
$$

Definition 6.3 (Product of primes within an interval.).

$$
p_{L} \sharp=\prod_{p_{k}<p_{i} \leq t}\left(p_{i}\right) \quad \text { s.t. } p_{i}=6 s+5 \quad p_{U} \sharp=\prod_{p_{k}<p_{i} \leq t}\left(p_{i}\right) \quad \text { s.t. } p_{i}=6 s+7
$$

The products of primes $p_{L} \sharp$ and $p_{U} \sharp$ : Definition 6.3 are defined to equal 1 , in the case that there are no pertinent primes within the interval. From the Definitions 2.4, 2.5 and inequality 3.1 we derive:

$$
\mathcal{L B}_{t} \leq \log \mathcal{M}_{t}=\log \left(\frac{\left(p_{k}+c\right) !}{\left(p_{k} ! \times c !\right)}\right)=\sum_{p \leq t} \mathcal{K}_{p} \log p \leq \mathcal{U B}_{t} \quad \forall p_{k} \in \mathbb{N} \mid p_{k} \geq 5
$$

Where $\mathrm{p}$ is as usual a prime number. The bounds on the logarithm of $\mathcal{M}_{L}$ we derive from Lemma 2.7 and 2.8 and the Definition 6.3 (case : LPF primes):

$$
\begin{aligned}
\mathcal{L B}_{L}= & \left(\log \left(\frac{(t+1)^{(t+1)}}{\left(p_{k}+1\right)^{\left(p_{k}+1\right)}(c+1)^{(c+1)}\left(p_{U} \sharp\right)}\right)\right) \\
\leq & \log \mathcal{M}_{L}=\sum_{k=1}^{c} \log \left(p_{k}+k\right)-\sum_{k=1}^{c} \log k-\sum_{p_{k}<p_{U}}^{t} \log p_{U} \\
\leq & \log \left(\frac{t^{t}}{p_{k}^{p_{k}} c^{c}\left(p_{U} \sharp\right)}\right)-1=\mathcal{U} \mathcal{B}_{L} \quad \forall p_{k} \in \mathbb{N} \mid p_{k} \geq 5
\end{aligned}
$$

The proof of the Solution Bound Theorem implements the Supremum bound function $\mathcal{U B}_{L s}$. This means that all AP UPF primes within the interval $p_{k}<p_{U} \leq t$ are factored out from $\log \mathcal{M}_{t}$ function and its bounds. Due to the fact that the Supremum function $\mathcal{U} \mathcal{B}_{L}$ applies values of $p_{k}, c$ and $t$ directly, it imposes a technical requirement to generate a set of pertinent values, to correctly approximate the interval $s$. This is to ascertain that the generated interval is at least equal to $s$, and the corresponding value of $c$ is correct. Respective definitions follow:

Definition 6.4.

$$
p_{k_{s}}=\frac{p_{k}}{2}
$$

\section{Definition 6.5.}

$$
c_{s}=\left\lfloor 5 \sqrt{\left(p_{k_{s}}\right)}\right\rfloor
$$




\section{Definition 6.6.}

$$
t_{s}=p_{k s}+c_{s}
$$

Theorem 6.7 (Bound on AP primes within an interval).

For any $p_{k} \in \mathbb{N} \mid p_{k} \geq 5$, there exists at least one $\boldsymbol{A P} L P F / U P F$ prime number $p_{j} \in \mathbb{N} \mid p_{k}<p_{j} \leq t$. The upper bound $\mathcal{U B}_{p_{k}}$ is given by:

$$
\begin{array}{r}
c=\mathcal{U} \mathcal{B}_{p_{k}}=\left\lfloor 5 \sqrt{p_{k}}\right\rfloor \quad \forall p_{k} \in \mathbb{N} \mid p_{k} \geq 5 \\
\text { Equivalently, } p_{j}-p_{k} \leq \mathcal{U B}_{\left(p_{k}\right)}
\end{array}
$$

Remark 6.1. The proof considers the AP LPF primes only. The case of AP UPF primes is analogous, thus it is left as an exercise for the reader (need to replace the $p_{U} \sharp$ with $p_{L} \sharp$ ).

Proof.

Suppose that there is no AP LPF prime within the interval from $p_{k}$ to $t$ for $p_{k} \in \mathbb{N} \mid p_{k} \geq 199$. Then in accordance with the hypothesis, by Lemma 3.1 we have that, every prime factor $p$ (since UPF primes were a priori factored out: the term $\left.p_{U} \sharp\right)$ of $\mathcal{M}_{(t)}$ must be less than or equal to $s=\left\lfloor\frac{t}{2}\right\rfloor$. Invoking Definitions $6.4,6.5$ and 6.6, the inequality 6.1 , we derive for all $p_{k} \in \mathbb{N} \mid p_{k} \geq 199$ :

$$
\begin{aligned}
& \text { (6.4) } \mathcal{L B}_{L}=\log \left(\frac{(t+1)^{(t+1)}}{\left(p_{k}+1\right)^{\left(p_{k}+1\right)}(c+1)^{(c+1)}\left(p_{U} \sharp\right)}\right) \\
& \leq \log \mathcal{M}_{L}=\sum_{p \leq t_{(s)}} \mathcal{K}_{p} \log p-\sum_{p_{k}<p_{U}}^{t} \log p_{U} \leq \log \left(\frac{\left(t_{s}\right)^{t_{s}}}{\left(p_{k_{s}}\right)^{p_{k_{s}}}\left(c_{s}\right)^{c_{s}}\left(p_{U} \sharp\right)}\right)-1=\mathcal{U} \mathcal{B}_{L_{s}}
\end{aligned}
$$

In accordance with the hypothesis therefore, it must be true that:

$$
a_{c}=\log \left(\frac{(t+1)^{(t+1)}}{\left(p_{k}+1\right)^{\left(p_{k}+1\right)}(c+1)^{(c+1)}}\right)-\log \left(\frac{\left(t_{s}\right)^{t_{s}}}{\left(p_{k_{s}}\right)^{p_{k_{s}}}\left(c_{s}\right)^{c_{s}}}\right)+1<0
$$

Now, we apply the Cauchy's Root Test for $p_{k} \geq 199$ :

$$
\lim _{c \rightarrow \infty} \sqrt[c]{\left|a_{c}\right|}=\lim _{c \rightarrow \infty} \sqrt[c]{\left|\mathcal{L B}_{L}-\mathcal{U} \mathcal{B}_{L_{s}}\right|}
$$

At $p_{k}=199$ the Cauchy's Root Test attains approx. 1.05921 and tends asymptotically to 1 , decreasing strictly from above. Thus, by the definition of the Cauchy's Root Test, the series formed from the terms of the difference $\mathcal{L} \mathcal{B}_{(t)}-\mathcal{U} \mathcal{B}_{\left(t_{s}\right)}$, diverges as $p_{k}$ increases unboundedly. Hence in accordance with the hypothesis, inequality 6.5 diverges to $-\infty$ as $p_{k}$ increases unboundedly. However, at $p_{k}=199$ the difference 6.5 attains approx. 56.0882 and diverges as $p_{k}$ increases unboundedly. Hence, we have a contradiction to the initial hypothesis. This implies that for all $p_{k} \in \mathbb{N} \mid p_{k} \geq 199$ :

$$
\mathcal{L B}_{t}-\mathcal{U B}_{t_{s}}>0
$$

Necessarily therefore, there must be at least one AP LPF prime within the interval $c$ for any $p_{k} \in \mathbb{N} \mid p_{k} \geq 199$. Table 4 (in the Appendix) lists all pertinent primes within the interval $p_{k} \in \mathbb{N} \mid 5 \leq p_{k} \leq 199, n \leq t$. Thus we deduce that Theorem 6.7 holds in this range as well. Consequently Theorem 6.7 holds as stated for all $p_{k} \in \mathbb{N} \mid p_{k} \geq 5$, hence completing the proof. 

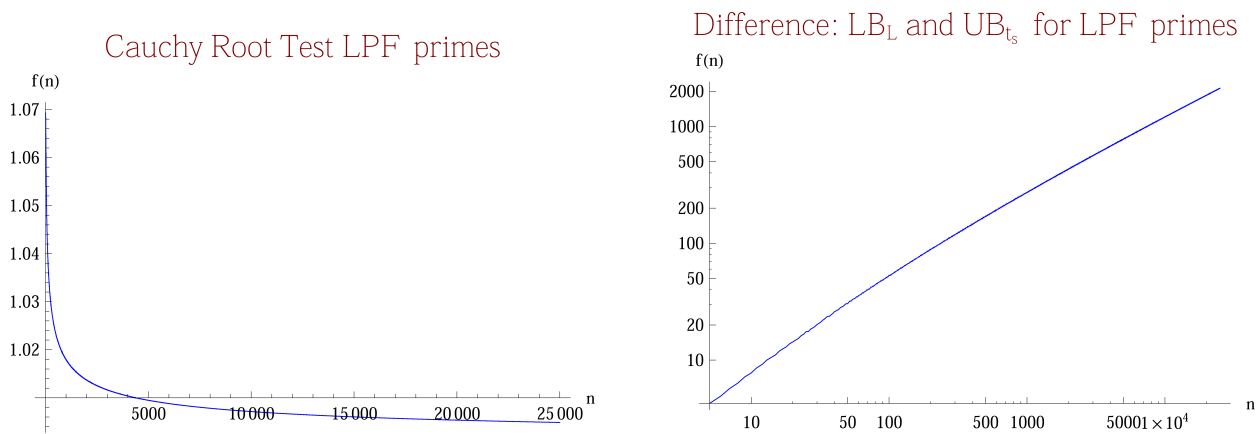

Figure 3. The L.H. drawing shows the Cauchy's Root Test. The R.H. log-log drawing shows the difference of the $\mathcal{L B}_{L}-\mathcal{U B}_{L_{s}}$. The figures are drawn at every $n \in \mathbb{N} \mid 5 \leq n \leq 25000$.

Definition 6.8. $c=n=e / 2$

Definition 6.9. $t=n+c=2 n$

Definition 6.10 (Product of primes in the interval.).

$$
p_{S} \sharp=\prod_{p_{i} \leq n}\left(p_{i}\right) \quad \text { for } p_{i}=\left(e-p_{j}\right), \text { and } p_{i} \leq p_{j}
$$

In the case that there are no prime summands in the interval, $p_{S} \sharp:=1$. It is necessary and sufficient for the product of primes $p_{S} \sharp$, to take into account the smaller prime of the solution pair only.

Theorem 6.11 (The Binary/Strong Goldbach Conjecture).

The exist at least one solution to $e=p_{i}+p_{j} \mid e, p_{i}, p_{j} \leq 2 n$ for $n \in \mathbb{N} \mid n=e / 2$ and $p_{i} \leq p_{j}$. This implies that any number $e \equiv 0(\bmod 2) \mid e \geq 4$ can be formed as a sum of two prime numbers. The combination of primes that comprise the solution is not unique.

Proof.

Suppose that for some $n \in \mathbb{N} \mid n \geq 227, n=e / 2$ there is no solution to $e=p_{i}+p_{j} \mid p_{i} \leq e / 2 \leq p_{j}$. From the Definitions 2.4, 2.5 and inequality 3.1, as well as Definitions 6.8 and 6.9 we derive for all $n \in \mathbb{N} \mid n \geq 227$ :

$$
\begin{aligned}
& \mathcal{L B}_{t}=\log \left(\frac{(t+1)^{(t+1)}}{(n+1)^{(n+1)}(c+1)^{(c+1)}}\right) \\
& \quad \leq \log \mathcal{M}_{t}=\sum_{p \leq t} \mathcal{K}_{p} \log p=\log \left(\frac{t !}{(n !)(c !)}\right)
\end{aligned}
$$

Since for all $n \in \mathbb{N} \mid n \geq 227$ we have $n>2(5 \sqrt{n})$, therefore by Theorem 6.7, multiple $p_{j}$ exist within the interval of length $c=n \mid n \leq p_{j}<t$. Thus necessarily, in accordance with the hypothesis, this implies that not a single $p_{i}=e-p_{j}$ exists. Consequently, in accordance with the Definition 6.10:

$$
p_{S} \sharp=\prod_{p_{j} \leq t}\left(e-p_{j}\right)=1
$$


From inequality 6.8 and equation 6.9 we have:

$$
a_{c}=\log \left(\frac{(t+1)^{(t+1)}}{(n+1)^{(n+1)}(c+1)^{(c+1)}}\right)-\log \left(\frac{t !}{(n !)(c !)\left(p_{S} \sharp\right)}\right)<0
$$

Now, we apply the Cauchy's Root Test :

$$
\lim _{c \rightarrow \infty} \sqrt[c]{\left|a_{c}\right|}=\lim _{c \rightarrow \infty} \sqrt[c]{\left|\mathcal{L B}_{t}-\log \left(\frac{t !}{(n !)(c !)\left(p_{S} \sharp\right)}\right)\right|}
$$

At $n=227$ the Cauchy's Root Test attains approx. 1.01722 and tends asymptotically to 1 , decreasing strictly from above. Please refer to Figure 5. Thus, by the definition of the Cauchy's Root Test, the sequence formed from the terms of the difference $\mathcal{L B}_{(t)}-\log \mathcal{M}_{t}$, diverges as $n$ increases unboundedly. Hence in accordance with the hypothesis, inequality 6.10 diverges to $-\infty$ as $n$ increases unboundedly. However, at $n=227$ the difference 6.10 attains approx. 42.8228 and diverges as $n$ increases unboundedly. Please also refer to Figure 5 . Hence, we have a contradiction to the initial hypothesis. This implies that for all $n \in \mathbb{N} \mid n \geq 227$ :

$$
\mathcal{L B}_{t}-\left(\log \mathcal{M}_{t}-\log \left(p_{S} \sharp\right)\right)>0
$$

Necessarily therefore, there must be at least one prime solution within the interval $c$ for all $n \in \mathbb{N} \mid n \geq 227$. Table 5 in the Appendix lists the number of solutions at any $e \in \mathbb{N} \mid 2 \leq e \leq 468$. Figure 4 shows the graph of the number of existing solutions at every even number within the range. Evidently, every possible even number $e \in \mathbb{N} \mid 4 \leq e \leq 468$ is satisfied. Thus we deduce that Theorem 6.11 holds in this range as well. Consequently Theorem 6.11 holds as stated for all $e \in \mathbb{N} \mid e \geq 4$, thus completing the proof.

\section{Goldbach: Number of solutions for e}

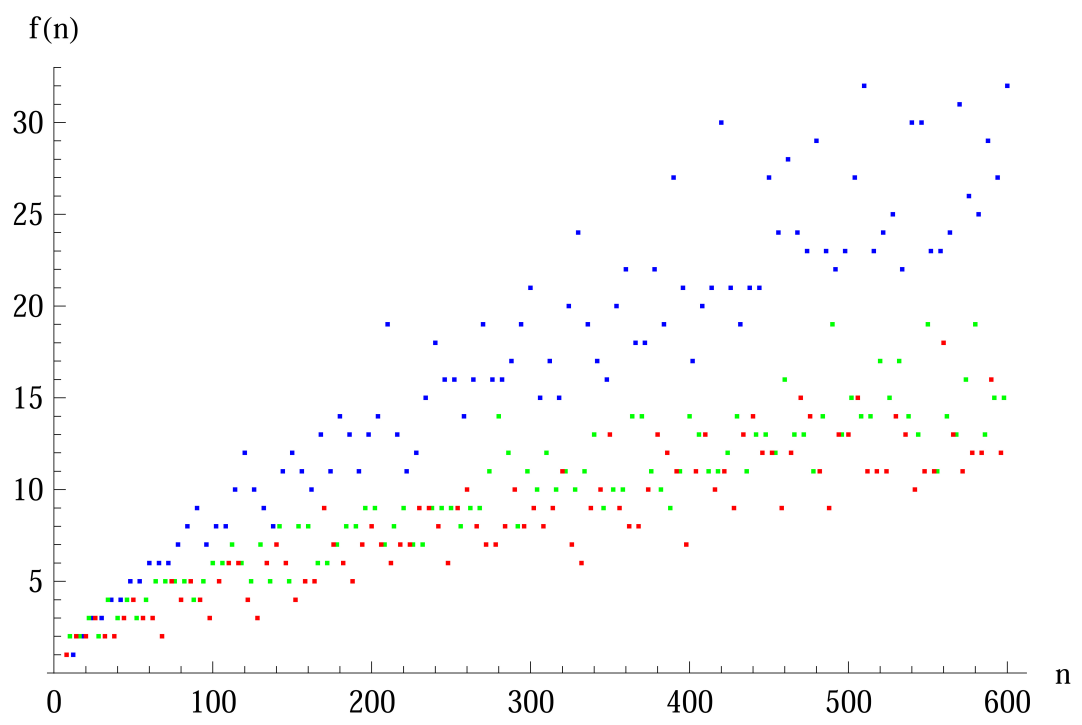

FIgURE 4. The drawing shows the number of existing solutions of $e=p_{i}+p_{j}$ at every even number $e \in \mathbb{N} \mid 8 \leq e \leq 600$. 

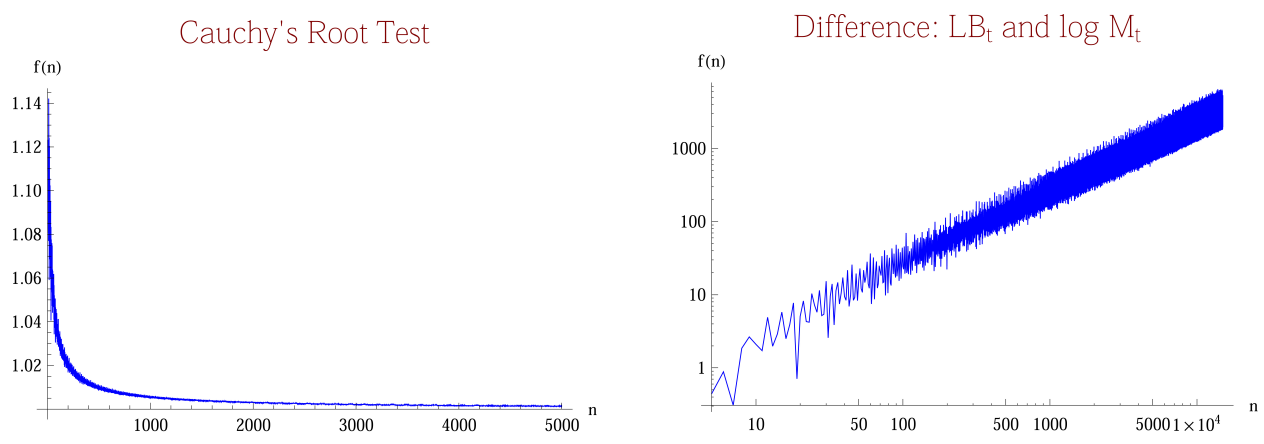

FiguRE 5. The L.H. drawing shows the Root Test. The R.H. log$\log$ drawing shows the difference 6.10. They are drawn at every $n \in \mathbb{N} \mid 5 \leq n \leq 15000$.

Figure 4 displays the so called Goldbach's comet. The Figure 6 in the Appendix shows an enhanced version of that graph. The color coding implemented in both Figures denotes:

- Even numbers $e \in \mathbb{N} \mid e=6 m+4$ comprised of All LPF solutions in Green.

- Even numbers $e \in \mathbb{N} \mid e=6 m+6$ comprised of Mixed solutions in Blue.

- Even numbers $e \in \mathbb{N} \mid e=6 m+8$ comprised of All UPF solutions in Red.

The number of all LPF or all UPF solutions to $e=p_{i}+p_{j}$ varies, it does so however within common for LPF/UPF boundaries. Clearly, the number of the Mixed solutions to $e=p_{i}+p_{j}$ exceeds that of the LPF or UPF solutions. The reason of that is that there are essentially two solution sets in the Mixed case. One set is comprised of solutions to $e=p_{i}+p_{j} \mid p_{i} \in L P F$, while $p_{j} \in U P F$ and the second set of solutions is $e=p_{i}+p_{j} \mid p_{i} \in U P F$, while $p_{j} \in L P F$. This at times results in almost doubling the solution set, while in the remaining instances it maintains the solution set significantly larger than either LPF/UPF. Hence creating the bands of solutions on the graph.

Corollary 6.12 (The Ternary Goldbach Conjecture).

The ternary Goldbach conjecture asserts that $\forall s \in \mathbb{N} \mid s \equiv 1(\bmod 2)$ and $s \geq 7$, $s$ can be written as the sum of three prime numbers.

Proof.

By Theorem 6.11 the Binary Goldbach Conjecture holds, for all $e \in \mathbb{N} \mid e \geq 4$. Therefore, any arbitrary integer $e \in \mathbb{N} \mid e \equiv 0(\bmod 2)$ and $e \geq 4$ can be written as a sum of two prime numbers. Corollary 6.12 clearly holds in the case of $p=7$ as $7=2+2+3$. Now, an arbitrary $s \in \mathbb{N} \mid s \equiv 1(\bmod 2), s>7$, may also be written as $s=e+3$. Because $e$ is an arbitrary even integer, which may be written as the sum of exactly two primes, necessarily therefore $s$, which is an arbitrary odd integer, may be written as the sum of three primes. Hence Corollary 6.12, holds as stated $\forall s \in \mathbb{N} \mid s \equiv 1(\bmod 2)$ and $s \geq 7$. 
Corollary 6.13 resolves a question posed by Shi et al [22],

Corollary 6.13 (Representation of Even Numbers by the Difference of 2 Primes).

Any even number $e \in \mathbb{N} \cup\{0\}$ represents the difference between two prime numbers.

Proof.

By Theorem 6.11 the Binary Goldbach Conjecture holds, for all $e \in \mathbb{N} \mid e \geq 4$. Therefore, any arbitrary integer $e \in \mathbb{N} \mid e \equiv 0(\bmod 2)$ can be written as:

$$
p_{i}+p_{j}=e=2 s, \forall s \in \mathbb{N} \mid s \geq 2 \text { and } p_{i}<p_{j}
$$

Obviously,

$$
\left(p_{i}+p_{j}\right)-p_{i}=p_{j}
$$

Consequently,

$$
\left(p_{i}+p_{j}\right)-2 p_{i}=p_{j}-p_{i}
$$

This implies that

$$
e-2 p_{i}=2 s-2 p_{i}=2\left(s-p_{i}\right)=p_{j}-p_{i}
$$

Which by Theorem 6.11 holds for any two prime numbers $p_{i}, p_{j} \in \mathbb{N} \mid p_{i}+p_{j}=e$ s.t. $e \in \mathbb{N} \mid e \equiv 0(\bmod 2), \quad e \geq 4$. In case of Twin Primes we have $\left(p_{j}+p_{i}\right)-$ $2\left(p_{i}\right)=2$, with $p_{j}=p_{i}+2$, while in the case of a double of any prime we have $\left(p_{j}+p_{i}\right)-2\left(p_{i}\right)=0$, with $p_{j}=p_{i}$. Thus Corollary 6.13 holds in these cases as well. Necessarily, we deduce that Corollary 6.13 holds as stated, concluding the proof.

From equations 4.4 through 4.6 , there are two cases to consider when contemplating the difference of two prime numbers. These cases generate two disjoint sets of even numbers. Firstly, all LPF or all UPF difference collapses into one case, while the Mixed case produces 2 instances;

- Case 1: The even number $e$ is all LPF or all UPF variety. The difference of two prime numbers $p_{i}, p_{j} \in \mathbb{N} \mid p_{i}<p_{j}$ produces an even integer of the form:

$$
e=(6 m+C)-(6 n+C)=6(m-n), \text { generating the set }\{6,12,18,24, \ldots\}
$$

- Case 2: The even number $e$ is of the Mixed variety. The sum of an arbitrary LPF integer and an arbitrary UPF integer produces an even integer of the form:

$$
\begin{aligned}
& e=\left(6 m+C_{2}\right)-\left(6 n+C_{1}\right)=6(m-n)-\left(C_{2}-C_{1}\right) \\
& \text { with }\left(C_{2}-C_{1}\right)= \pm 2, \text { generating the set }\{4,8,10,14, \ldots\}
\end{aligned}
$$

It is obvious that the union of the two solution sets given above 6.17 and 6.18 , produces the whole set of the even integers $e \in \mathbb{N} \mid e \geq 4$. 


\section{REFERENCES}

[1] Vigo Brun, Le crible d'Erastothene et le theoreme de Goldbach, C. R. Acad. Sci. Paris $\mathbf{1 6 8}$ (1919), 544-546.

[2] Elena Calude, The Complexity of Goldbachs Conjecture and Riemanns Hypothesis, CDMTCS Research, Massey University Albany, NZ (2009).

[3] Avery Carr, The Strong Goldbach Conjecture: An Equivalent Statement, AMS Blog, https://blogs.ams.org/mathgradblog/2013/09/18/equivalent-statement-strong-Goldbachconjecture (2013).

[4] J. Chen, On the representation of a large even integer as the sum of a prime and the product of at most 2 primes, Kexue Tongbao 17 (1966), 385-386.

[5] J. Deshouillers, G. Effinger, H. Te Riele, and D. Zinoviev, A complete Vinogradov 3-primes theorem under the Riemann hypothesis, Electronic Research Announcements of the American Mathematical Society 15 (1997), 99-104.

[6] Apostolos Doxiadis, Uncle Petros and Goldbach's Conjecture, Faber and Faber, 2000.

[7] Paul Erdös, On the difference of consecutive primes, Quarterly Journal Of Mathematics (1935).

[8] Paul Erdös and E.G. Strauss, Remarks on the differences between consecutive primes, Elem. Math. 35 (1980), 115-118.

[9] Jan Feliksiak, The elementary proof of the Riemann's Hypothesis, MDPI, AG, https://doi.org/10.20944/preprints202006.0365.v1 (2020).

[10] - The maximal prime gaps Supremum and the Firoozbakht's Hypothesis No 30, MDPI, AG, https://doi.org/10.20944/preprints202006.0366.v1 (2020).

[11] _ Maximal prime gaps bounds, ScienceOpen, Inc, https://doi.org/10.14293/S21991006.1.SOR-.PPWVKRR.v1 (2021).

[12] _ The Brocard Conjecture, ScienceOpen, Inc, https://doi.org/10.14293/S21991006.1.SOR-.PPIH4LV.v1 (2021).

[13] G.H. Hardy and E.M. Wright, An introduction to the theory of numbers, Oxford University Press, London, 1968.

[14] G.H. Hardy and J.E. Littlewood, Some problems of Partitio numerorum, On the expression of a number as a sum of primes, Acta Mathematica 44 (1923), 1-70.

[15] D.R. Heath-Brown, Differences between consecutive primes, Jahresber. Deutsch. Mathem. Ver. 90 (1988), 71-89.

[16] Leszek Kaniecki, On Schnirelman's constant under the Riemann hypothesis, Acta Arithmetica 4 (1995), 361-374.

[17] Edmund Landau, Vorlesungen über Zahlentheorie, Vol. II, S. Hirzel, Leipzig, 1927.

[18] János Pintz, Very large gaps between consecutive primes, Journal Of Number Theory (1997).

[19] Robert A. Rankin, The difference between consecutive prime numbers, Journal London Mathematical Society (1938)

[20] Atle Selberg, On the normal density of primes in small intervals and the difference between consecutive primes, Arch. Mathem. B 47 (1943), 87-105.

[21] An elementary proof of the prime number theorem, Ann. Math. 50 (1949), 305-313.

[22] Honwei Shi, Zhou Mi, Delong Zhang, Xingyi Jiang, and Songting He, Even numbers are the sum of two prime numbers, Greener Journal of Science GJSETR 9 (1) (2019), 8-11.

[23] Daniel Shanks, On maximal gaps between successive primes, Math. Comp. 18 (1964), 646651.

[24] Tomas Oliveira e Silva, Gaps between consecutive primes, 2006. www.ieeta.pt/ tos/gaps.html.

[25] K. Soundararajan, Small gaps between prime numbers: the work of Goldston-Pintz-Yildirim, Bulletin of the American Mathematical Society (2007).

[26] I.M. Vinogradov, Some theorems in analytic theory of numbers, Dokl. Akad. Nauk SSSR 4 (1934), 185-187.

[27] Yuan Wang, Goldbach conjecture, World Scientific Publishing N.J., 2000.

[28] E. Westzynthius, Über die Differenzen Verteilung der Zahlen die zu den $n$ ersten Primzahlen teilerfremd sind, Comm. Phys. Math. Soc. Sci. Fenn. 5 (1931), 1-37. 


\section{Appendix}

The tabulated data concerns the Theorems 6.7 and 6.11 respectively.

TABle 4. Bound on AP Primes: Theorem 6.7

\begin{tabular}{|c|c|l|}
\hline \multicolumn{3}{|c|}{ The Set of Primes within interval $\mathcal{U B}_{p_{k}}$} \\
\hline$p_{k}$ & $\mathcal{U B}_{p_{k}}$ & Set of LPF Primes \\
\hline 5 & 11 & $\{11\}$ \\
\hline 7 & 13 & $\{11,17\}$ \\
\hline 11 & 16 & $\{17,23\}$ \\
\hline 13 & 18 & $\{17,23,29\}$ \\
\hline 17 & 20 & $\{23,29\}$ \\
\hline 19 & 21 & $\{23,29\}$ \\
\hline 23 & 23 & $\{29,41\}$ \\
\hline 29 & 26 & $\{41,47,53\}$ \\
\hline 31 & 27 & $\{41,47,53\}$ \\
\hline 37 & 30 & $\{41,47,53,59\}$ \\
\hline 41 & 32 & $\{47,53,59,71\}$ \\
\hline 43 & 32 & $\{47,53,59,71\}$ \\
\hline 47 & 34 & $\{53,59,71\}$ \\
\hline 53 & 36 & $\{59,71,83,89\}$ \\
\hline 59 & 38 & $\{71,83,89\}$ \\
\hline 61 & 39 & $\{71,83,89\}$ \\
\hline 67 & 40 & $\{71,83,89,101,107\}$ \\
\hline 71 & 42 & $\{83,89,101,107,113\}$ \\
\hline 73 & 42 & $\{83,89,101,107,113\}$ \\
\hline 79 & 44 & $\{83,89,101,107,113\}$ \\
\hline 83 & 45 & $\{89,101,107,113\}$ \\
\hline 89 & 47 & $\{101,107,113,131\}$ \\
\hline 97 & 49 & $\{101,107,113,131,137\}$ \\
\hline 101 & 50 & $\{107,113,131,137,149\}$ \\
\hline 103 & 50 & $\{107,113,131,137,149\}$ \\
\hline 107 & 51 & $\{113,131,137,149\}$ \\
\hline 109 & 52 & $\{113,131,137,149\}$ \\
\hline 113 & 53 & $\{131,137,149\}$ \\
\hline 127 & 56 & $\{131,137,149,167,173,179\}$ \\
\hline 131 & 57 & $\{137,149,167,173,179\}$ \\
\hline 137 & 58 & $\{149,167,173,179,191\}$ \\
\hline 139 & 58 & $\{149,167,173,179,191,197\}$ \\
\hline 149 & 61 & $\{167,173,179,191,197\}$ \\
\hline 151 & 61 & $\{167,173,179,191,197\}$ \\
\hline 157 & 62 & $\{167,173,179,191,197\}$ \\
\hline 163 & 63 & $\{167,173,179,191,197\}$ \\
\hline & & \\
\hline & & \\
\hline
\end{tabular}


TABLE 4. Continued

\begin{tabular}{|l|c|l|}
\hline \multicolumn{4}{|c|}{ The Set of Primes within the interval $\mathcal{U B}_{p_{k}}$ (Continued) } \\
\hline 167 & 64 & $\{173,179,191,197,227\}$ \\
\hline 173 & 65 & $\{179,191,197,227,233\}$ \\
\hline 179 & 66 & $\{191,197,227,233,239\}$ \\
\hline 181 & 67 & $\{191,197,227,233,239\}$ \\
\hline 191 & 69 & $\{197,227,233,239,251,257\}$ \\
\hline 193 & 69 & $\{197,227,233,239,251,257\}$ \\
\hline 197 & 70 & $\{227,233,239,251,257,263\}$ \\
\hline 199 & 70 & $\{227,233,239,251,257,263,269\}$ \\
\hline \multicolumn{3}{|c|}{ The End } \\
\hline
\end{tabular}

TABLE 5. The Binary Goldbach Conjecture: Theorem 6.11

\begin{tabular}{|c|c|c|c|c|c|}
\hline \multicolumn{5}{|c|}{ Number of solutions of $e=p_{i}+p_{j}$} & \multirow{2}{*}{$\frac{e \equiv 0(\bmod 2)}{\text { Solutions }}$} \\
\hline $\bar{e}$ & Solutions & $e$ & Solutions & $e$ & \\
\hline 4 & 1 & 158 & 5 & 314 & 9 \\
\hline 6 & 1 & 160 & 8 & 316 & 10 \\
\hline 8 & 1 & 162 & 10 & 318 & 15 \\
\hline 10 & 2 & 164 & 5 & 320 & 11 \\
\hline 12 & 1 & 166 & 6 & 322 & 11 \\
\hline 14 & 2 & 168 & 13 & 324 & 20 \\
\hline 16 & 2 & 170 & 9 & 326 & 7 \\
\hline 18 & 2 & 172 & 6 & 328 & 10 \\
\hline 20 & 2 & 174 & 11 & 330 & 24 \\
\hline 22 & 3 & 176 & 7 & 332 & 6 \\
\hline 24 & 3 & 178 & 7 & 334 & 11 \\
\hline 26 & 3 & 180 & 14 & 336 & 19 \\
\hline 28 & 2 & 182 & 6 & 338 & 9 \\
\hline 30 & 3 & 184 & 8 & 340 & 13 \\
\hline 32 & 2 & 186 & 13 & 342 & 17 \\
\hline 34 & 4 & 188 & 5 & 344 & 10 \\
\hline 36 & 4 & 190 & 8 & 346 & 9 \\
\hline 38 & 2 & 192 & 11 & 348 & 16 \\
\hline 40 & 3 & 194 & 7 & 350 & 13 \\
\hline 42 & 4 & 196 & 9 & 352 & 10 \\
\hline 44 & 3 & 198 & 13 & 354 & 20 \\
\hline 46 & 4 & 200 & 8 & 356 & 9 \\
\hline 48 & 5 & 202 & 9 & 358 & 10 \\
\hline 50 & 4 & 204 & 14 & 360 & 22 \\
\hline 52 & 3 & 206 & 7 & 362 & 8 \\
\hline 54 & 5 & 208 & 7 & 364 & 14 \\
\hline 56 & 3 & 210 & 19 & 366 & 18 \\
\hline 58 & 4 & 212 & 6 & 368 & 8 \\
\hline & & & tinued & & \\
\hline
\end{tabular}


TABle 5. Continued

\begin{tabular}{|c|c|c|c|c|c|}
\hline \multicolumn{6}{|c|}{ Solutions (Continued) } \\
\hline 60 & 6 & 214 & 8 & 370 & 14 \\
\hline 62 & 3 & 216 & 13 & 372 & 18 \\
\hline 64 & 5 & 218 & 7 & 374 & 10 \\
\hline 66 & 6 & 220 & 9 & 376 & 11 \\
\hline 68 & 2 & 222 & 11 & 378 & 22 \\
\hline 70 & 5 & 224 & 7 & 380 & $\overline{13}$ \\
\hline 72 & 6 & 226 & 7 & 382 & 10 \\
\hline 74 & 5 & \begin{tabular}{|l|l}
228 \\
\end{tabular} & 12 & 384 & 19 \\
\hline 76 & 5 & 230 & 9 & 386 & 12 \\
\hline 78 & 7 & 232 & 7 & 388 & 9 \\
\hline 80 & 4 & 234 & 15 & 390 & 27 \\
\hline 82 & 5 & 236 & 9 & 392 & 11 \\
\hline 84 & 8 & 238 & 9 & 394 & 11 \\
\hline $\begin{array}{l}86 \\
\end{array}$ & 5 & 240 & 18 & 396 & 21 \\
\hline 88 & 4 & 242 & 8 & 398 & 7 \\
\hline 90 & 9 & 244 & 9 & 400 & $\overline{14}$ \\
\hline $\begin{array}{l}92 \\
\end{array}$ & 4 & \begin{tabular}{|l|l}
246 \\
\end{tabular} & 16 & 402 & 17 \\
\hline 94 & 5 & 248 & 6 & 404 & 11 \\
\hline 96 & 7 & 250 & 9 & 406 & 13 \\
\hline 98 & 3 & 252 & 16 & 408 & 20 \\
\hline 100 & 6 & 254 & 9 & 410 & 13 \\
\hline 102 & 8 & 256 & 8 & 412 & 11 \\
\hline 104 & 5 & 258 & 14 & 414 & 21 \\
\hline 106 & 6 & 260 & 10 & 416 & 10 \\
\hline 108 & 8 & 262 & 9 & 418 & 11 \\
\hline 110 & 6 & 264 & 16 & 420 & 30 \\
\hline 112 & 7 & 266 & 8 & 422 & 11 \\
\hline 114 & 10 & 268 & 9 & 424 & 12 \\
\hline 116 & 6 & 270 & 19 & 426 & 21 \\
\hline 118 & 6 & 272 & 7 & 428 & 9 \\
\hline 120 & 12 & 274 & 11 & 430 & 14 \\
\hline 122 & 4 & 276 & 16 & 432 & 19 \\
\hline 124 & 5 & 278 & 7 & 434 & $\overline{13}$ \\
\hline 126 & 10 & 280 & 14 & 436 & 11 \\
\hline 128 & 3 & 282 & 16 & 438 & 21 \\
\hline 130 & 7 & 284 & 8 & 440 & $\overline{14}$ \\
\hline 132 & 9 & 286 & 12 & 442 & 13 \\
\hline 134 & 6 & 288 & 17 & 444 & 21 \\
\hline 136 & 5 & 290 & 10 & 446 & 12 \\
\hline 138 & 8 & 292 & 8 & 448 & 13 \\
\hline 140 & 7 & 294 & 19 & 450 & 27 \\
\hline 142 & 8 & 296 & 8 & 452 & 12 \\
\hline 144 & 11 & 298 & 11 & 454 & 12 \\
\hline
\end{tabular}


Table 5. Continued

\begin{tabular}{|c|c|c|c|c|c|}
\hline \multicolumn{7}{|c|}{ Solutions (Continued) } \\
\hline 146 & 6 & 300 & 21 & 456 & 24 \\
\hline 148 & 5 & 302 & 9 & 458 & 9 \\
\hline 150 & 12 & 304 & 10 & 460 & 16 \\
\hline 152 & 4 & 306 & 15 & 462 & 28 \\
\hline 154 & 8 & 308 & 8 & 464 & 12 \\
\hline 156 & 11 & 310 & 12 & 466 & 13 \\
\hline- & - & 312 & 17 & 468 & 24 \\
\hline \multicolumn{7}{|c|}{ The End } \\
\hline
\end{tabular}


